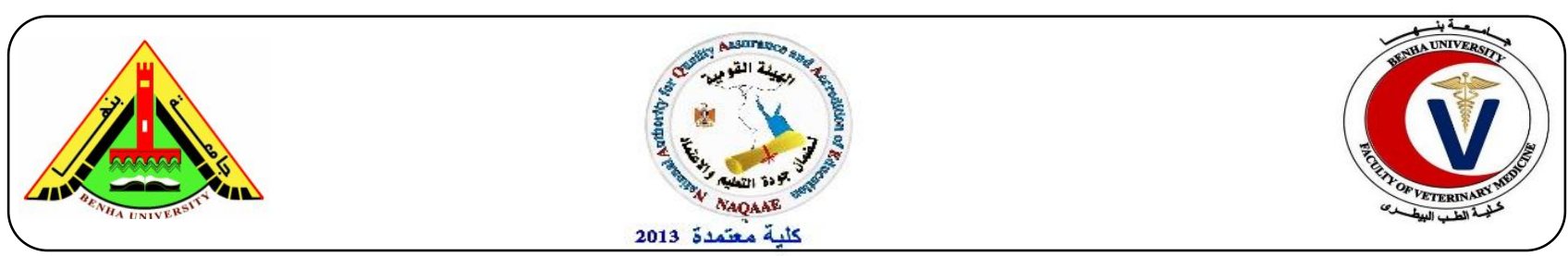

\title{
Biochemical effects of Pomegranate Grinded Peels on High Sucrose Stressed Rabbits
}

\author{
Abdelmaksoud H.A. ${ }^{1}$, Omnia, M. Abdelhamid ${ }^{1}$, Taheya, E.A. Ismaeel ${ }^{2}$, Waheed A. Elkharadly ${ }^{1}$ \\ ${ }^{1}$ Department of Biochemistry, Faculty of Veterinary Medicine, Benha University \\ ${ }^{2}$ Department of Nutrition and clinical nutrition, Faculty of Veterinary Medicine, Benha University
}

\section{A B S T R A C T}

Pomegranate is a drought tolerant and long lived plant which grows popularly in arid and semiarid zones in the Mediterranean countries. Pomegranate was reported to have antimicrobial, antiinflammatory, anti-tumor, anti-hepatotoxic, antiviral, anti-diabetic activities and they can improve oral, skin and cardiovascular health. Sucrose is a non-reducing disaccharide made up of 50\% glucose and $50 \%$ fructose and has a moderately high glycemic index. High-sucrose diets have been used in animal models to induce well-characterized metabolic dysfunctions including cardiovascular risk factors, insulin resistance, dyslipidemia and hypertension. The present research aimed to evaluate the effects of pomegranate grinded peel extract supplementation on blood parameters of male rabbits stressed by high sucrose diet for 4 months, Through evaluation of serum total lipids, triacylglycerols, total cholesterol, HDL-c, LDL-c, VLDL-c and atherogenic indices, in addition to serum urea, creatinine, glucose, testosterone, zinc and copper concentrations. High sucrose supplementation induced a significant increase in Serum total lipids, triacylglycerols, total cholesterol, LDL-c, VLDL-c, atherogenic indices, glucose, testosterone and copper concentrations, in addition to non-significant increase in serum urea, creatinine concentrations. On contrast, it exhibited a significant decrease in serum HDL-C and zinc concentrations. Pomegranate peel powder supplementation was able to mitigate and ameliorate stress induced by high sucrose ration and showed pronounced curative effect against hyperlipidemia and deviated serum atherogenic indices as well as maintained glucose, testosterone copper and zinc toward control levels. The results of the present study suggested that pomegranate has the potential to exert curative effects against hyperlipidemia.

Keywords: Pomegranate, Sucrose, Hyperlipidemia, Oxidative stress.

(http://www.bvmj.bu.edu.eg)

(BVMJ-35(1): 152-163, 2018)

\section{INTRODUCTION}

Pomegranate (Punica granatum) is used for the treatment of various sicknesses because of its bioactive compounds, antimicrobial, antiinflammatory, anti-tumor, anti-hepatotoxic, antiviral, anti-diabetic activities and they can improve oral, skin and cardiovascular health. Biological actions of pomegranate fruit that lead it to be considered as a healthy fruit are due to its potent phytochemicals contents that 
scavenge wide spectrum of free radicals (Seyedeh et al., 2017).

The pomegranate peel is rich in polyphenolic compounds resembling tannins (punicalagin pedunculagan, gallagic acid, ellagic acid and its esters of glucose and flavonoids (Sarker and Gohda, 2013). Moreover, Lansky and Newman, (2007) reported that the powerful phytochemicals contents of pomegranate fruit are responsible for its biological actions that lead it to be considered as a healthy fruit and act as scavenger of wide spectrum of free radicals.

The anti-obesity effect of the pomegranate leaves extract after oral administration is linked to acting as appetite suppressant; it also acts as pancreatic lipase inhibitor in obese mice (Lei et al., 2007). The advantages of the pomegranate have been linked to the tannins increase weight loss fat oxidation and total antioxidant status and decrease serum glucose and insulin lipid peroxidation serum cholesterol oxidative stress and cholesterol: HDL-C ratio so PLE prevent and protect against diabetes obesity hypertension and cardiovascular disease Bialonska et al (2010) Ellagic acid has antimutagenic, antioxidant, and anti- inflammatory activity in bacterial and mammalian cell. It is indicated by epidemiological studies, that the incidence of coronary heart diseases is inversely proportional to the intake of the fruits due to the phenolic compounds antioxidant action of ellagic acid (Indria et al., 2000).

Pomegranate fruit contains many phenolic compounds with high antioxidant activity, which may have useful health properties including flavonoids anthocyanins and hydrolysable tannins which attribuated to nearby $92 \%$ of pomegranate antioxidant activity, where the main component of pomegranate husk is Punicalagin, which is one of polyphenols of pomegranateare agents that have the ability for restraining ROS effect on the body and have protective activities against inflammation, cancer and atherosclerosis (Lee et al., 2008).

Pomegranate extract has double anti-obesity effects through inhibiting activity of lipase in addition to suppressing intake of energy. Also the pomegranate extract and Sibutramine are used in health center as an appetite suppressant have the same works where the calorie intake of rat fed an HFD has been decreased markedly by the pomegranate extract in the same way but not of those fed a normal diet (Lei et al., 2007).

Sucrose is a none reducing disaccharide made up of $50 \%$ glucose and $50 \%$ fructose and has a moderately high glycemic index of 80 , about the same as pasteurized processed honey, 75, and more than raw honey, 35, (Healthy Sugar Alternatives, 2012). Most adverse effects of sucrose are related to its content of fructose (Watson et al., 2003). Rats fed a high-fructose diet (>60\% of total calories) provide a cluster of abnormalities, which include hypertension, hypertriglyceridemia and glucose intolerance in addition to hyper-insulinemia which induce hypertension, hypertriglyceridemia, increased collagen deposition in the heart and kidneys associated with increased oxidant concentrations and decreased antioxidant defenses (Kamari et al., 2008).

In this respect, fructose, as a component of sucrose, is a major ingredient of many processed foods and contributed to the development of obesity and dyslipidemia in humans (Bray, 2010). Indeed, diets rich in this sugar induce hepatic steatosis and plasma hyperlipidemia in rodents (Anuradha and Anurag, 2002).

Chronic exposure to fructose causes hyperinsulinaemia and obesity through altered mechanisms that include the effect of fructose on ATP depletion and uric acid generation and increasing circulating C-peptide levels that are often associated with insulin resistance (Johnson et al., 2010), involvement 
of the fructose transporter shows significantly higher expression levels in obese rats compared to lean controls and the hexosamine hypothesis, in which hexosamine flux is thought to be involved in regulating glucose pathways (Litherland et al., 2004).

Aim of the work: Studying the biochemical role of pomegranate on high sucrose stressed rabbits.

\section{MATERIALS AND METHODS}

\subsection{Ration and additives:}

Animals were fed on a basal ration throughout 14 days acclimatization period of the experiment in the form of pelleted concentrated ration shown in the following table (1):

\begin{tabular}{|c|c|c|c|c|c|}
\hline 1 & Carbohydrates & $58 \%$ & 5 & Minerals & $\begin{array}{c}1.49 \\
\%\end{array}$ \\
\hline 2 & Protein & $\begin{array}{c}20.5 \\
\%\end{array}$ & 6 & Calcium & $\begin{array}{c}0.98 \\
\%\end{array}$ \\
\hline 3 & Lipid & $\begin{array}{c}3.4 \\
\%\end{array}$ & 7 & Phosphorus & $\begin{array}{c}0.53 \\
\%\end{array}$ \\
\hline 4 & Cellulose & $\begin{array}{c}3.1 \\
\%\end{array}$ & 8 & Moisture & $12 \%$ \\
\hline \multicolumn{6}{|c|}{ Total } \\
\hline
\end{tabular}

\subsection{Pomegranate peel:}

After gathering fresh Pomegranate from fruit farm in Cairo-Alexandria desert road at the region of Borg El-Arab (Egypt), Fresh peel were carefully cleaned and washed by distilled water and dried, then were powdered and passed through mesh to increase their contact with powdered ration.

\subsection{Chemical composition of Pomegranate} peel: Table (2)

\begin{tabular}{|c|l|c|l|l|c|}
\hline 1 & Moisture & $\begin{array}{c}13.70 \\
\%\end{array}$ & 5 & Fiber & $\begin{array}{c}11.22 \\
\%\end{array}$ \\
\hline 2 & Protein & $\begin{array}{c}3.10 \\
\%\end{array}$ & 6 & $\begin{array}{l}\text { Total } \\
\text { phenolic }\end{array}$ & $\begin{array}{c}27.90 \\
\%\end{array}$ \\
\hline 3 & Carbohydrates & $\begin{array}{c}39.05 \\
\%\end{array}$ & 7 & Ash & $\begin{array}{c}3.30 \\
\%\end{array}$ \\
\hline 4 & Fat & $\begin{array}{c}1.73 \\
\%\end{array}$ & \multicolumn{3}{c|}{ Total $100 \%$} \\
\hline \multicolumn{7}{|c|}{} \\
\hline
\end{tabular}

Then the powder sent to ration manufacture to be pellet as $25 \%$ of the ration ratio according to Maha (2017) as following: Table (3)

\begin{tabular}{|c|l|c|c|l|c|}
\hline 1 & $\begin{array}{l}\text { Pomegranate } \\
\text { peel powder }\end{array}$ & $\begin{array}{c}25 \\
\%\end{array}$ & 6 & Minerals & $\begin{array}{c}1.49 \\
\%\end{array}$ \\
\hline 2 & Carbohydrates & $\begin{array}{c}38 \\
\%\end{array}$ & 7 & Calcium & $\begin{array}{c}0.98 \\
\%\end{array}$ \\
\hline 3 & Protein & $\begin{array}{c}15.5 \\
\%\end{array}$ & 8 & Phosphorus & $\begin{array}{c}0.53 \\
\%\end{array}$ \\
\hline 4 & Lipid & $\begin{array}{c}3.4 \\
\%\end{array}$ & 9 & Moisture & $12 \%$ \\
\hline 5 & Cellulose & $\begin{array}{c}3.1 \\
\%\end{array}$ & & \\
\hline \multicolumn{7}{|c|}{ Total $100 \%$} \\
\hline
\end{tabular}

\subsection{Feed additives:}

Sucrose is powdered and mixed with ration in $25 \%$ concentration of the ration then pelleted according to (El-Tabbakh, 2014). Table (4)

\begin{tabular}{|c|l|c|c|l|c|}
\hline 1 & Sucrose & $25 \%$ & 6 & Minerals & $\begin{array}{c}1.49 \\
\%\end{array}$ \\
\hline 2 & Carbohydrates & $38 \%$ & 7 & Calcium & $\begin{array}{c}0.98 \\
\%\end{array}$ \\
\hline 3 & Protein & $\begin{array}{c}15.5 \\
\%\end{array}$ & 8 & Phosphorus & $\begin{array}{c}0.53 \\
\%\end{array}$ \\
\hline 4 & Lipid & $3.4 \%$ & 9 & Moisture & $12 \%$ \\
\hline 5 & Cellulose & $3.1 \%$ & & & \\
\hline \multicolumn{5}{|c|}{ Total $100 \%$} \\
\hline
\end{tabular}

\subsection{Experimental animals:}

A total number of eighty (80) male rabbits at age of 4 weeks old after winning and weighting about 500-600 gm, were used in the experimental investigation of this study, and obtained from the Laboratory Animals Research Center, Fac. Vet. Med., Benha University, rabbits were housed in separated metal cages, exposed to good ventilation, humidity and to a 12-hr light-dark cycle, and provided with a constant supply of standard pellet diet and fresh, clean drinking water ad libitum.

\subsection{Experimental design:}

Rabbits were allocated into four groups of 20 rabbits in each, placed in individual cages and classified as following: (Group 1): 20 rabbits, served as control normal group, kept on basal ration only for 4 months; (Group 2): 20 rabbits, served as sucrose stressed group, 
reared on the $25 \%$ sucrose concentration ration for 4 months; (Group 3): 20 rabbits, reared on $25 \%$ pomegranate peel powder ration only for 4 months; (Group 4): 20 rabbits, reared on equal amounts of $25 \%$ sucrose and pomegranate peel concentration powdered rations for 4 months.

\subsection{Sampling:}

Blood samples were collected from all animals monthly for four months, the first one after one month (from ear veins), the second after 2 months, the third sample after third month then the last after 4 months. Blood samples were collected in tubes without anticoagulant in clean, dry Wassermann tubes and left in slope position to clot at room temperature. The tubes were centrifuged at $3000 \mathrm{rpm}$ for 5 minutes and the non hemolyzed serum was carefully separated and transferred into clean dry eppendorf tube which kept frozen at $-20^{\circ} \mathrm{C}$ until used for biochemical analysis.

\subsection{Determination of serum biochemical} markers:

Total lipids (Zöllner and Krish.,1962), Triacylglycerols (Stein, 1987), Total cholesterol (Searcy, 1969), HDL-C (Burstein et al., 1970), LDL-C (Friedewald et al., 1972), VLDL-C (Bauer, 1982), Atherogenic indices (Cardiac Risk Ratio (CRR), Atherogenic Index (AI), Atherogenic Coefficient (AC) (Dobiásová, 2004), Urea (Kaplan et al., 1984), Creatinine (Schirmeister et al.,1984), Glucose (Caraway and Watts, 1987), Testosterone (Rommerts, 2004), Zinc (Johnsen and Eliasson 1987), Copper (Abe et al., 1989).

\subsection{Statistical analysis:}

The results were expressed as mean $( \pm$ S.E. $)$ and statistical significance was evaluated by one way ANOVA using SPSS (version 10.0) program followed by the post hoc test, least significant difference (LSD). Values were considered statistically significant when $\mathrm{p}<0.05$.

\section{RESULTS}

The obtained data in Tables $(5,6)$ revealed that high sucrose ration exhibited a significant increase in serum total lipids, triacylglycerols, total cholesterol, LDL-c, VLDL-c concentrations and serum atherogenic indices, in addition to serum glucose, testosterone and copper concentrations, accompanied with a non-significant increase in serum urea, creatinine concentrations. On contrast, it exhibited a significant decrease in serum HDL-C and zinc concentrations in sucrose stressed rabbits, when compared with control group.

Administration of pomegranate peel powder as treatment to high sucrose stressed group, resulted in significant decreases in serum total lipids, triacylglycerols, total cholesterol, LDL-c, VLDL-c concentrations and serum atherogenic indices, in addition to serum glucose, testosterone and copper concentrations, accompanied with a significant decrease in serum urea, creatinine concentrations. On contrast, it exhibited a significant increase in serum HDL-C and zinc concentrations, in comparison with sucrose stressed group.

The obtained data in Tables $(5,6)$ revealed that, supplementation of pomegranate peel powder only to normal rabbits exhibited a significant increase in serum total lipids, triacylglycerols, VLDL-c concentrations and serum atherogenic indices, in addition to serum zinc and copper concentrations. On contrast, it exhibited a significant decrease in serum total cholesterol, HDL-C, LDL-C, urea, creatinine glucose, testosterone and concentrations, in comparison with control normal group. 
Table (5): Effect of pomegranate peel powder treatment on blood biochemical parameters in experimental sucrose induced stress in male rabbits:

\begin{tabular}{|c|c|c|c|c|c|c|c|c|c|c|}
\hline & $\begin{array}{l}\text { Animal } \\
\text { groups }\end{array}$ & $\begin{array}{l}\text { Total } \\
\text { lipids } \\
\mathrm{mg} / \mathrm{dl}\end{array}$ & $\begin{array}{l}\mathrm{TAG} \\
\mathrm{mg} / \mathrm{dl}\end{array}$ & $\begin{array}{l}\text { T.Choles } \\
\mathrm{mg} / \mathrm{dl}\end{array}$ & $\begin{array}{l}\text { HDL- } \\
\text { C } \\
\mathrm{mg} / \mathrm{dl}\end{array}$ & $\begin{array}{c}\text { LDL- } \\
\text { C } \\
\mathrm{mg} / \mathrm{dl}\end{array}$ & $\begin{array}{l}\text { VLDL- } \\
\text { C } \\
\mathrm{mg} / \mathrm{dl}\end{array}$ & CRR & AI & $\mathrm{AC}$ \\
\hline \multirow{4}{*}{$\begin{array}{c}\text { After } \\
1^{\text {st }} \\
\text { month }\end{array}$} & $\begin{array}{l}\text { Control } \\
\text { normal }\end{array}$ & $\begin{array}{l}223.2 \\
\pm 5.33^{\mathrm{d}}\end{array}$ & $\begin{array}{c}33.97 \\
\pm \\
1.73^{\mathrm{d}}\end{array}$ & $\begin{array}{l}45.84 \\
\pm 3.24^{\mathrm{b}}\end{array}$ & $\begin{array}{c}26.64 \\
\pm \\
0.83^{\mathrm{a}}\end{array}$ & $\begin{array}{c}13.14 \\
\pm \\
2.99^{\mathrm{ab}}\end{array}$ & $\begin{array}{c}6.79 \\
\pm 0.35^{\mathrm{d}}\end{array}$ & $\begin{array}{c}1.42 \\
\pm \\
0.13^{\mathrm{c}}\end{array}$ & $\begin{array}{c}0.10 \\
\pm \\
0.03^{\mathrm{c}}\end{array}$ & $\begin{array}{c}0.76 \\
\pm \\
0.13^{\mathrm{c}}\end{array}$ \\
\hline & $\begin{array}{l}\text { Sucrose } \\
\text { induced stress }\end{array}$ & $\begin{array}{l}350.28 \\
\pm 8.20^{\mathrm{a}}\end{array}$ & $\begin{array}{l}149.31 \\
\pm 5.04^{\mathrm{a}}\end{array}$ & $\begin{array}{c}59.13 \\
\pm 4.72^{\mathrm{a}}\end{array}$ & $\begin{array}{c}11.34 \\
\pm \\
1.85^{\mathrm{c}}\end{array}$ & $\begin{array}{c}14.40 \\
\pm \\
0.80^{\mathrm{ab}}\end{array}$ & $\begin{array}{r}29.86 \\
\pm 3.60^{\mathrm{a}}\end{array}$ & $\begin{array}{c}4.69 \\
\pm \\
0.81^{\mathrm{a}}\end{array}$ & $\begin{array}{c}0.96 \\
\pm \\
0.09^{\mathrm{a}}\end{array}$ & $\begin{array}{c}3.79 \\
\pm \\
0.81^{\mathrm{a}}\end{array}$ \\
\hline & $\begin{array}{l}\text { Pomegranate } \\
\text { only }\end{array}$ & $\begin{array}{l}249.64 \\
\pm 5.94^{c}\end{array}$ & $\begin{array}{r}51.93 \\
\pm 4.69^{\mathrm{c}}\end{array}$ & $\begin{array}{r}35.88 \\
\pm 1.29^{\mathrm{c}}\end{array}$ & $\begin{array}{c}14.04 \\
\pm \\
14.04^{\mathrm{c}}\end{array}$ & $\begin{array}{c}10.34 \\
\pm \\
1.83^{\mathrm{b}}\end{array}$ & $\begin{array}{r}19.65 \\
\pm 2.90^{c}\end{array}$ & $\begin{array}{c}1.62 \\
\pm \\
1.00^{\mathrm{c}}\end{array}$ & $\begin{array}{c}0.80 \\
\pm \\
0.02^{\mathrm{b}}\end{array}$ & $\begin{array}{c}0.81 \\
\pm \\
0.09^{\mathrm{c}}\end{array}$ \\
\hline & $\begin{array}{l}\text { Sucrose }+ \\
\text { Pomegranate }\end{array}$ & $\begin{array}{l}298.64 \\
\pm 7.65^{\mathrm{b}}\end{array}$ & $\begin{array}{c}97.59 \\
\pm \\
3.61^{b}\end{array}$ & $\begin{array}{c}53.44 \\
\pm 2.07^{\mathrm{ab}}\end{array}$ & $\begin{array}{c}21.06 \\
\pm \\
21.06^{\mathrm{b}}\end{array}$ & $\begin{array}{c}12.32 \\
\pm \\
1.81^{\mathrm{a}}\end{array}$ & $\begin{array}{c}22.52 \\
\pm 0.72^{\mathrm{b}}\end{array}$ & $\begin{array}{c}2.36 \\
\pm \\
0.24^{\mathrm{b}}\end{array}$ & $\begin{array}{c}0.74 \\
\pm \\
0.03^{\mathrm{b}}\end{array}$ & $\begin{array}{c}1.63 \\
\pm \\
0.24^{\mathrm{b}}\end{array}$ \\
\hline \multirow{4}{*}{$\begin{array}{l}\text { After } \\
2^{\text {nd }} \\
\text { month }\end{array}$} & $\begin{array}{l}\text { Control } \\
\text { normal }\end{array}$ & $\begin{array}{l}230.04 \\
\pm 4.48^{\mathrm{d}}\end{array}$ & $\begin{array}{c}* 48.15 \\
\pm \\
1.80^{\mathrm{d}}\end{array}$ & $\begin{array}{l}54.00 \\
\pm 3.87^{\mathrm{b}}\end{array}$ & $\begin{array}{c}24.32 \\
\pm \\
22.32^{\mathrm{b}}\end{array}$ & $\begin{array}{c}13.80 \\
\pm \\
2.53^{\mathrm{b}}\end{array}$ & $\begin{array}{l}9.63 \\
\pm 0.36^{\mathrm{d}}\end{array}$ & $\begin{array}{c}2.23 \\
\pm \\
0.29^{\mathrm{b}}\end{array}$ & $\begin{array}{c}0.30 \\
\pm \\
0.01^{\mathrm{c}}\end{array}$ & $\begin{array}{c}1.33 \\
\pm \\
0.29^{\mathrm{b}}\end{array}$ \\
\hline & $\begin{array}{l}\text { Sucrose } \\
\text { induced stress }\end{array}$ & $\begin{array}{l}366.84 \\
\pm 6.75^{\mathrm{a}}\end{array}$ & $\begin{array}{c}* \\
218.57 \\
\pm 7.88^{\mathrm{a}}\end{array}$ & $\begin{array}{l}64.92 \\
\pm 3.24^{\mathrm{a}}\end{array}$ & $\begin{array}{c}10.07 \\
\pm \\
0.90^{\mathrm{c}}\end{array}$ & $\begin{array}{c}* 21.42 \\
\pm \\
2.17^{\mathrm{a}}\end{array}$ & $\begin{array}{l}* 43.71 \\
\pm 2.78^{\mathrm{a}}\end{array}$ & $\begin{array}{c}4.37 \\
\pm \\
0.30^{\mathrm{a}}\end{array}$ & $\begin{array}{c}1.09 \\
\pm \\
0.02^{\mathrm{a}}\end{array}$ & $\begin{array}{c}3.47 \\
\pm \\
0.30^{\mathrm{a}}\end{array}$ \\
\hline & $\begin{array}{l}\text { Pomegranate } \\
\text { only }\end{array}$ & $\begin{array}{l}261.58 \\
\pm 5.11^{\mathrm{c}}\end{array}$ & $\begin{array}{r}96.52 \\
\pm 4.50^{c}\end{array}$ & $\begin{array}{l}* 49.50 \\
\pm 2.71^{\mathrm{c}}\end{array}$ & $\begin{array}{c}* 21.06 \\
\pm \\
1.49^{\mathrm{b}}\end{array}$ & $\begin{array}{c}11.48 \\
\pm \\
1.26^{\mathrm{b}}\end{array}$ & $\begin{array}{r}21.67 \\
\pm 0.78^{c}\end{array}$ & $\begin{array}{c}2.23 \\
\pm \\
0.27^{\mathrm{b}}\end{array}$ & $\begin{array}{c}0.65 \\
\pm \\
0.02^{\mathrm{b}}\end{array}$ & $\begin{array}{c}1.78 \\
\pm \\
0.27^{\mathrm{b}}\end{array}$ \\
\hline & $\begin{array}{l}\text { Sucrose }+ \\
\text { Pomegranate }\end{array}$ & $\begin{array}{l}314.14 \\
\pm 7.90^{\mathrm{b}}\end{array}$ & $\begin{array}{c}154.24 \\
\pm \\
3.94^{\mathrm{b}}\end{array}$ & $\begin{array}{c}54.51 \\
\pm 2.83^{\mathrm{ab}}\end{array}$ & $\begin{array}{c}* 22.62 \\
\pm \\
1.10^{\mathrm{a}}\end{array}$ & $\begin{array}{c}18.22 \\
\pm \\
0.99^{\mathrm{a}}\end{array}$ & $\begin{array}{c}27.85 \\
\pm 0.79^{\mathrm{b}}\end{array}$ & $\begin{array}{c}1.79 \\
\pm \\
0.11^{\mathrm{c}}\end{array}$ & $\begin{array}{c}0.67 \\
\pm \\
0.02^{\mathrm{b}}\end{array}$ & $\begin{array}{c}0.89 \\
\pm \\
0.11^{\mathrm{c}}\end{array}$ \\
\hline \multirow{4}{*}{$\begin{array}{l}\text { After } \\
3^{\text {rd }} \\
\text { month }\end{array}$} & $\begin{array}{l}\text { Control } \\
\text { normal }\end{array}$ & $\begin{array}{l}266.08 \\
\pm 3.72^{\mathrm{d}}\end{array}$ & $\begin{array}{c}86.58 \\
\pm \\
1.04^{\mathrm{bc}}\end{array}$ & $\begin{array}{c}65.40 \\
\pm 2.75^{\mathrm{ab}}\end{array}$ & $\begin{array}{c}18.90 \\
\pm \\
1.65^{\mathrm{a}}\end{array}$ & $\begin{array}{c}14.18 \\
\pm \\
1.81^{\mathrm{a}}\end{array}$ & $\begin{array}{c}17.31 \\
\pm 1.00^{\mathrm{d}}\end{array}$ & $\begin{array}{c}3.21 \\
\pm \\
0.42^{\mathrm{b}}\end{array}$ & $\begin{array}{c}0.60 \\
\pm \\
0.02^{\mathrm{b}}\end{array}$ & $\begin{array}{c}2.31 \\
\pm \\
0.43^{\mathrm{b}}\end{array}$ \\
\hline & $\begin{array}{l}\text { Sucrose } \\
\text { induced stress }\end{array}$ & $\begin{array}{l}383.76 \\
\pm 6.08^{\mathrm{a}}\end{array}$ & $\begin{array}{l}206.65 \\
\pm 2.38^{\mathrm{a}}\end{array}$ & $\begin{array}{c}70.04 \\
\pm 1.70^{\mathrm{a}}\end{array}$ & $\begin{array}{c}8.64 \\
\pm \\
0.97^{\mathrm{b}}\end{array}$ & $\begin{array}{c}15.40 \\
\pm \\
1.53^{\mathrm{a}}\end{array}$ & $\begin{array}{c}39.33 \\
\pm 0.47^{\mathrm{a}}\end{array}$ & $\begin{array}{c}7.65 \\
\pm \\
88.20^{\mathrm{a}}\end{array}$ & $\begin{array}{c}1.23 \\
\pm \\
0.03^{\mathrm{a}}\end{array}$ & $\begin{array}{c}6.75 \\
\pm \\
0.88^{\mathrm{a}}\end{array}$ \\
\hline & $\begin{array}{l}\text { Pomegranate } \\
\text { only }\end{array}$ & $\begin{array}{l}299.36 \\
\pm 8.30^{\mathrm{c}}\end{array}$ & $\begin{array}{r}74.70 \\
\pm 1.80^{\mathrm{c}}\end{array}$ & $\begin{array}{c}57.89 \\
\pm 1.94^{\mathrm{c}}\end{array}$ & $\begin{array}{c}15.66 \\
\pm \\
1.22^{\mathrm{a}}\end{array}$ & $\begin{array}{c}12.78 \\
\pm \\
1.22^{\mathrm{a}}\end{array}$ & $\begin{array}{c}25.90 \\
\pm 0.37^{\mathrm{c}}\end{array}$ & $\begin{array}{c}3.77 \\
\pm \\
0.36^{\mathrm{c}}\end{array}$ & $\begin{array}{c}0.63 \\
\pm \\
0.03^{\mathrm{b}}\end{array}$ & $\begin{array}{c}2.97 \\
\pm \\
0.36^{\mathrm{b}}\end{array}$ \\
\hline & $\begin{array}{l}\text { Sucrose + } \\
\text { Pomegranate }\end{array}$ & $\begin{array}{l}336.68 \\
\pm 4.46^{\mathrm{b}}\end{array}$ & $\begin{array}{c}117.40 \\
\pm \\
3.71^{\mathrm{b}}\end{array}$ & $\begin{array}{c}64.05 \\
\pm 1.66^{\mathrm{ab}}\end{array}$ & $\begin{array}{c}19.04 \\
\pm \\
1.36^{\mathrm{a}}\end{array}$ & $\begin{array}{c}12.50 \\
\pm \\
0.90^{\mathrm{a}}\end{array}$ & $\begin{array}{c}32.48 \\
\pm 0.74^{\mathrm{b}}\end{array}$ & $\begin{array}{c}3.15 \\
\pm \\
0.27^{\mathrm{b}}\end{array}$ & $\begin{array}{c}0.54 \\
\pm \\
0.04^{\mathrm{bc}}\end{array}$ & $\begin{array}{c}2.25 \\
\pm \\
0.27^{\mathrm{b}}\end{array}$ \\
\hline \multirow[t]{2}{*}{$\begin{array}{c}\text { After } \\
4^{\text {th }} \\
\text { month }\end{array}$} & $\begin{array}{l}\text { Control } \\
\text { normal }\end{array}$ & $\begin{array}{l}291.28 \\
\pm 4.05^{\mathrm{d}}\end{array}$ & $\begin{array}{c}94.37 \\
\pm \\
1.13^{\mathrm{d}}\end{array}$ & $\begin{array}{l}71.28 \\
\pm 2.99^{\mathrm{ab}}\end{array}$ & $\begin{array}{c}20.60 \\
\pm \\
1.79^{\mathrm{a}}\end{array}$ & $\begin{array}{c}14.71 \\
\pm \\
1.97^{\mathrm{a}}\end{array}$ & $\begin{array}{c}18.86 \\
\pm 1.09^{\mathrm{b}}\end{array}$ & $\begin{array}{c}3.49 \\
\pm \\
0.45^{\mathrm{bc}}\end{array}$ & $\begin{array}{c}0.65 \\
\pm \\
0.02^{\mathrm{b}}\end{array}$ & $\begin{array}{c}2.51 \\
\pm \\
046^{\mathrm{bc}}\end{array}$ \\
\hline & Sucrose & 418.29 & 254.34 & 76.34 & 7.91 & 17.69 & 42.86 & 8.33 & 1.34 & 7.35 \\
\hline
\end{tabular}




\begin{tabular}{|c|c|c|c|c|c|c|c|c|c|}
\hline induced stress & $\pm 6.62^{\mathrm{a}}$ & $\pm 2.59^{\mathrm{a}}$ & $\pm 1.85^{\mathrm{a}}$ & $\begin{array}{c} \pm \\
1.05^{\mathrm{b}}\end{array}$ & $\begin{array}{c} \pm \\
1.66^{\mathrm{a}}\end{array}$ & $\pm 0.51^{\mathrm{a}}$ & $\begin{array}{c} \pm \\
96.13^{\mathrm{a}}\end{array}$ & $\begin{array}{c} \pm \\
0.03^{\mathrm{a}}\end{array}$ & $\begin{array}{c} \pm \\
0.95^{\mathrm{a}}\end{array}$ \\
\hline $\begin{array}{l}\text { Pomegranate } \\
\text { only }\end{array}$ & $\begin{array}{l}333.55 \\
\pm 9.04^{\mathrm{c}}\end{array}$ & $\begin{array}{l}121.42 \\
\pm 1.96^{\mathrm{c}}\end{array}$ & $\begin{array}{l}63.10 \\
\pm 2.11^{\mathrm{c}}\end{array}$ & $\begin{array}{c}17.06 \\
\pm \\
1.32^{\mathrm{a}}\end{array}$ & $\begin{array}{c}13.93 \\
\pm \\
1.32^{\mathrm{a}}\end{array}$ & $\begin{array}{c}26.33 \\
\pm \\
0.40^{\mathrm{bc}}\end{array}$ & $\begin{array}{c}4.10 \\
\pm \\
0.39^{\mathrm{b}}\end{array}$ & $\begin{array}{c}0.68 \\
\pm \\
0.03^{\mathrm{b}}\end{array}$ & $\begin{array}{c}3.32 \\
\pm \\
0.39^{\mathrm{b}}\end{array}$ \\
\hline $\begin{array}{l}\text { Sucrose + } \\
\text { Pomegranate }\end{array}$ & $\begin{array}{l}368.26 \\
\pm 4.86^{\mathrm{b}}\end{array}$ & $\begin{array}{c}149.36 \\
\pm \\
4.04^{\mathrm{b}}\end{array}$ & $\begin{array}{c}69.81 \\
\pm 1.80^{\mathrm{ab}}\end{array}$ & $\begin{array}{c}20.75 \\
\pm \\
1.48^{\mathrm{a}}\end{array}$ & $\begin{array}{c}13.62 \\
\pm \\
0.98^{\mathrm{a}}\end{array}$ & $\begin{array}{r}33.87 \\
\pm 0.80^{\mathrm{c}}\end{array}$ & $\begin{array}{c}3.43 \\
\pm \\
0.29^{\mathrm{bc}}\end{array}$ & $\begin{array}{c}0.58 \\
\pm \\
0.04^{\mathrm{bc}}\end{array}$ & $\begin{array}{c}2.45 \\
\pm \\
0.29^{\mathrm{bc}}\end{array}$ \\
\hline
\end{tabular}

Data are presented as (Mean \pm S.E), S.E $=$ Standard error. Mean values with different superscript letters in the same column are significantly different at $(\mathrm{P}<0.05)$.* Means a significant difference between periods of treatment $(\mathrm{P}<0.05)$.

Table (6): Effect of pomegranate peel powder treatment on blood biochemical parameters in experimental sucrose induced stress in male rabbits:

\begin{tabular}{|c|c|c|c|c|c|c|c|}
\hline & $\begin{array}{l}\text { Animal } \\
\text { groups }\end{array}$ & $\begin{array}{l}\text { Urea } \\
\mathrm{mg} / \mathrm{dl}\end{array}$ & $\begin{array}{c}\text { Creatinine } \\
\mathrm{mg} / \mathrm{dl}\end{array}$ & $\begin{array}{l}\text { Glucose } \\
\mathrm{mg} / \mathrm{dl}\end{array}$ & $\begin{array}{c}\text { Testosterone } \\
\mathrm{ng} / \mathrm{ml}\end{array}$ & $\begin{array}{l}\text { Zinc } \\
\mu \mathrm{g} / \mathrm{dl}\end{array}$ & $\begin{array}{l}\text { Copper } \\
\mu \mathrm{g} / \mathrm{dl}\end{array}$ \\
\hline \multirow{5}{*}{$\begin{array}{c}\text { After } \\
1^{\text {st }} \\
\text { month }\end{array}$} & Control normal & $\begin{array}{c}22.50 \pm \\
1.60^{\mathrm{a}}\end{array}$ & $\begin{array}{c}0.59 \pm \\
0.03^{\mathrm{a}}\end{array}$ & $\begin{array}{l}78.07 \pm \\
2.15^{\mathrm{b}}\end{array}$ & $1.23 \pm 0.04^{b}$ & $\begin{array}{c}164.47 \pm \\
2.21^{\mathrm{b}}\end{array}$ & $\begin{array}{c}22.05 \pm \\
1.48^{\mathrm{b}}\end{array}$ \\
\hline & Sucrose induced & $\begin{array}{c}22.95 \pm \\
0.58^{\mathrm{a}}\end{array}$ & $0.63 \pm$ & $91.26 \pm$ & $1.45 \pm 0.13^{\mathrm{a}}$ & $131.02 \pm$ & $32.22 \pm$ \\
\hline & (11000 & $184+$ & $056+$ & 2.11 & $0.91 \pm$ & $17977+$ & 1.78 \\
\hline & Pomegranate only & $0.93^{\mathrm{b}}$ & $0.02^{\mathrm{a}}$ & $1.50^{\mathrm{c}}$ & $0.03 b^{c}$ & $1.85^{\mathrm{a}}$ & $0.99^{\mathrm{b}}$ \\
\hline & $\begin{array}{l}\text { Sucrose + } \\
\text { Pomegranate }\end{array}$ & $\begin{array}{c}17.32 \pm \\
1.48^{\mathrm{b}}\end{array}$ & $\begin{array}{c}0.38 \pm \\
0.02^{\mathrm{b}}\end{array}$ & $\begin{array}{l}75.11 \pm \\
2.66^{\mathrm{b}}\end{array}$ & $1.08 \pm 0.02^{\mathrm{c}}$ & $\begin{array}{c}146.87 \pm \\
2.78^{\mathrm{c}}\end{array}$ & $\begin{array}{c}30.09 \pm \\
0.97^{\mathrm{a}}\end{array}$ \\
\hline \multirow{4}{*}{$\begin{array}{l}\text { After } \\
2^{\text {nd }} \\
\text { month }\end{array}$} & Control normal & $\begin{array}{c}27.00 \pm \\
0.97^{\mathrm{ab}}\end{array}$ & $\begin{array}{c}0.54 \pm \\
0.03^{\mathrm{a}}\end{array}$ & $\begin{array}{l}84.64 \pm \\
2.73^{\mathrm{b}}\end{array}$ & $1.67 \pm 0.17^{b}$ & $\begin{array}{c}171.67 \pm \\
4.05^{\mathrm{b}}\end{array}$ & $\begin{array}{l}25.71 \pm \\
0.97^{\mathrm{c}}\end{array}$ \\
\hline & $\begin{array}{l}\text { Sucrose induced } \\
\text { stress }\end{array}$ & $\begin{array}{c}30.60 \pm \\
1.32^{\mathrm{a}}\end{array}$ & $\begin{array}{c}0.61 \pm \\
0.05^{\mathrm{a}}\end{array}$ & $\begin{array}{c}120.15 \pm \\
1.87^{\mathrm{a}}\end{array}$ & $1.98 \pm 0.25^{\mathrm{a}}$ & $\begin{array}{c}145.27 \pm \\
5.67^{\mathrm{d}}\end{array}$ & $\begin{array}{c}43.40 \pm \\
1.23^{\mathrm{a}}\end{array}$ \\
\hline & Pomegranate only & $\begin{array}{c}23.40 \pm \\
1.28^{\mathrm{bc}}\end{array}$ & $\begin{array}{c}0.51 \pm \\
0.05^{\mathrm{a}}\end{array}$ & $\begin{array}{l}78.75 \pm \\
16.44^{\mathrm{c}}\end{array}$ & $0.96 \pm 0.06^{\mathrm{c}}$ & $\begin{array}{c}185.40 \pm \\
2.52^{\mathrm{a}}\end{array}$ & $\begin{array}{l}34.65 \pm \\
0.68^{\mathrm{b}}\end{array}$ \\
\hline & $\begin{array}{l}\text { Sucrose + } \\
\text { Pomegranate }\end{array}$ & $\begin{array}{r}20.47 \pm \\
1.61^{\mathrm{c}}\end{array}$ & $\begin{array}{c}0.45 \pm \\
0.03^{b}\end{array}$ & $\begin{array}{l}97.98 \pm \\
2.18^{\mathrm{b}}\end{array}$ & $1.11 \pm 0.05^{\mathrm{c}}$ & $\begin{array}{c}158.37 \pm \\
2.58^{\mathrm{c}}\end{array}$ & $\begin{array}{c}40.32 \pm \\
0.54^{\mathrm{a}}\end{array}$ \\
\hline \multirow{4}{*}{$\begin{array}{l}\text { After } \\
3^{\text {rd }} \\
\text { month }\end{array}$} & Control normal & $\begin{array}{c}28.80 \pm \\
0.97^{\mathrm{a}}\end{array}$ & $\begin{array}{c}0.69 \pm \\
0.07^{\mathrm{a}}\end{array}$ & $\begin{array}{c}93.60 \pm \\
2.79^{c}\end{array}$ & $1.87 \pm 0.22^{b}$ & $\begin{array}{c}190.50 \pm \\
5.70^{\mathrm{b}}\end{array}$ & $\begin{array}{c}27.77 \pm \\
2.51^{\mathrm{c}}\end{array}$ \\
\hline & $\begin{array}{l}\text { Sucrose induced } \\
\text { stress }\end{array}$ & $\begin{array}{c}29.70 \pm \\
1.32^{\mathrm{a}}\end{array}$ & $\begin{array}{c}0.72 \pm \\
0.03^{\mathrm{a}}\end{array}$ & $\begin{array}{c}143.05 \pm \\
1.49^{\mathrm{a}}\end{array}$ & $2.88 \pm 0.36^{\mathrm{a}}$ & $\begin{array}{c}153.35 \pm \\
3.75^{\mathrm{d}}\end{array}$ & $\begin{array}{c}45.90 \pm \\
1.51^{\mathrm{a}}\end{array}$ \\
\hline & Pomegranate only & $\begin{array}{c}24.30 \pm \\
1.68^{\mathrm{b}}\end{array}$ & $\begin{array}{c}0.65 \pm \\
0.01^{\mathrm{a}}\end{array}$ & $\begin{array}{c}81.00 \pm \\
3.03^{\mathrm{d}}\end{array}$ & $0.98 \pm 0.03^{\mathrm{c}}$ & $\begin{array}{c}202.17 \pm \\
4.95^{\mathrm{b}}\end{array}$ & $\begin{array}{c}46.60 \pm \\
1.12^{\mathrm{a}}\end{array}$ \\
\hline & $\begin{array}{l}\text { Sucrose + } \\
\text { Pomegranate }\end{array}$ & $\begin{array}{c}23.40 \pm \\
1.42^{\mathrm{b}}\end{array}$ & $\begin{array}{c}0.63 \pm \\
0.03^{b}\end{array}$ & $\begin{array}{c}110.32 \pm \\
2.85^{\mathrm{b}}\end{array}$ & $1.01 \pm 0.05^{\mathrm{c}}$ & $\begin{array}{c}166.20 \pm \\
6.25^{\mathrm{c}}\end{array}$ & $\begin{array}{c}43.65 \pm \\
2.02^{\mathrm{a}}\end{array}$ \\
\hline \multirow{4}{*}{$\begin{array}{c}\text { After } \\
4^{\text {th }} \\
\text { month }\end{array}$} & Control normal & $\begin{array}{c}29.31 \pm \\
1.05^{\mathrm{a}}\end{array}$ & $\begin{array}{c}0.75 \pm \\
0.07^{\mathrm{a}}\end{array}$ & $\begin{array}{c}102.02 \pm \\
3.04^{\mathrm{c}}\end{array}$ & $1.79 \pm 0.32^{\mathrm{b}}$ & $\begin{array}{c}210.91 \pm \\
6.21^{\mathrm{b}}\end{array}$ & $\begin{array}{c}30.23 \pm \\
2.00^{\mathrm{c}}\end{array}$ \\
\hline & $\begin{array}{l}\text { Sucrose induced } \\
\text { stress }\end{array}$ & $\begin{array}{c}32.37 \pm \\
1.41^{\mathrm{a}}\end{array}$ & $\begin{array}{c}0.78 \pm \\
0.03^{\mathrm{a}}\end{array}$ & $\begin{array}{c}162.32 \pm \\
1.62^{\mathrm{a}}\end{array}$ & $2.95 \pm 0.33^{\mathrm{a}}$ & $\begin{array}{c}162.15 \pm \\
4.08^{\mathrm{d}}\end{array}$ & $\begin{array}{c}52.12 \pm \\
1.98^{\mathrm{a}}\end{array}$ \\
\hline & Pomegranate only & $\begin{array}{c}26.48 \pm \\
1.83^{\mathrm{b}}\end{array}$ & $\begin{array}{c}0.70 \pm \\
0.01^{\mathrm{b}}\end{array}$ & $\begin{array}{c}88.29 \pm \\
3.30^{\mathrm{d}}\end{array}$ & $1.27 \pm 0.25^{\mathrm{c}}$ & $\begin{array}{c}211.64 \pm \\
5.39^{\mathrm{a}}\end{array}$ & $\begin{array}{c}49.13 \pm \\
3.00^{\mathrm{a}}\end{array}$ \\
\hline & Sucrose + & $25.50 \pm$ & $0.68 \pm$ & $127.54 \pm$ & $0.99 \pm 0.30^{c}$ & $181.85 \pm$ & $48.67 \pm$ \\
\hline
\end{tabular}




\begin{tabular}{|c|c|c|c|}
\hline & Pomegranate & 1.17 & $0.03^{b}$ \\
\hline
\end{tabular}

\section{DISCUSSION}

The aim of this experimental study was to evaluate the potential benefit of pomegranate peel powder supplementation on sucrose stressed rabbits, on some biochemical parameters levels.

The obtained data in table 5 showed that, the high sucrose feeding significantly increased serum total lipids, Tg and TC in all periods, the increasing effect still after withdrawal time also. This was agree with (El-Tabakh, 2014) who report that, the increment in total lipids may attributed to Fructose which has been shown to increase plasma concentrations of triacylglycerols and cholesterol.

In this respect, fructose, as a component of sucrose, is a major ingredient of many processed foods and has been proposed to contribute to the development of obesity and dyslipidemia in humans (Gross et al., 2004). Indeed, diets rich in this sugar induce hepatic steatosis and plasma hyperlipidemia in rodents (Anuradha and Anurag, 2002). In this context a short-term consumption of a highsucrose diet increases the triglyceride levels in liver and in plasma (Busserolles et al., 2002b). Moreover, Hypertriglyceridemia after simple carbohydrate feeding, such as fructose and/or sucrose, results from the induction of DeNovo lipogenesis, the enhanced rate of hepatic VLDL-triglyceride synthesis and a decrease in peripheral triglyceride clearance (Suzuki and Hara 2004).

The obtained results revealed that, the high sucrose plus Pomegranate peel lead to significant decrease in serum total lipid, triglycerides and total cholesterol in compared to group fed with high sucrose diet only. The decreasing effect still after withdrawal time also. This came in accordance with the data of Paniagua et al., (2007) who mentioned that, the pomegranate peel diet prevents central body fat accumulation and decreases postprandial adiponectin expression induced by a carbohydrate rich diet in insulin-resistant subjects. And studies show a direct beneficial role for olive oil in improving plasma lipids in the treatment of metabolic syndrome (Alonso et al., 2006). Also Pomegranate peel improved insulin resistance, increased the release of TG from the liver and decreased the flux of FFAs from peripheral adipose tissue back to the liver (Maha, 2017) who reported that, the administration of pomegranate peel resulted in reductions of total cholesterol concentration and transaminases activity in serum of the oleuropein group compared to ethanol-treated rats.

The present data reported that, the high sucrose feeding significantly decrease the serum HDL-C in group fed on high sucrose diet in comparing with others at all in three periods of the experiment. Furthermore, high sucrose diet significantly increases LDL-c after 60 days of treatment. These results agree with Maurya and Srivastava (2008) reported that, glucose intolerance and insulin resistance is associated with dyslipidemia characterized by a high TG in test group, significant high levels of TC, LDL-C, non-esterified fatty acid and glycerol in high fructose diet hamsters with a significant low level of HDL-c. In addition in subjects consuming fructose, significantly increased circulating levels of remnant lipoproteins, small dense LDL, and oxidized LDL (Stanhope et al., 2007).

The obtained data also revealed that pomegranate peel significantly increase serum HDL-c after 60 and 120 days of treatment and after withdrawal time also, this may be explained in studies which suggest that an oxidation reaction is involved in small, dense 
LDL formation of Small, dense LDL are more prone to oxidation and to enter into the arterial wall more readily than larger buoyant LDL particles, thus, accelerating the development of atherosclerosis (Lee et al., 2008).

In addition, the particle size of the LDL lipoprotein is influenced by the dietary fat. Low-fat diets lead to a decrease in the mean LDL size compared to high-fat diets. However, increase the LDL particle size more than a carbohydrate-rich diet, this effect being influenced by the apo E genotypes. Moreover Pomegranate peel been shown to be less susceptible to oxidation. This binding is directly related to an increase of the LDL resistance to oxidation (Lan et al., 2009). These results agree with (Sarker and Gohda, 2013) who reported that, intake of Pomegranate peel is associated with significantly increased HDL-cholesterol concentrations and PON activity.

The postulated data were agree with Salwe-kartik et al., (2015) who found that pomegranate peel decreasing LDL-c and the anti-atherosclerotic effect of Pomegranate peel ex $\neg$ tract was also demonstrated in rabbits on a high-lipid diet. As Animals in the highlipid diet group had higher levels of TC, TAG, and LDL cholesterol, as well as a thick layer of lipid disposition in the aortic intema. These results support pomegranate peel has anti-atherosclerotic effect, most likely related to suppression of inflammation. Similarly the Pomegranate peel extract decreased the concentration of TG and LDL-cholesterol and improved the level of HDL-cholesterol that has been linked to a lower risk of coronary heart disease (Rosenblat et al. 2006).

These results reported that, high sucrose diet has no significant effect on blood urea and serum creatinine level in compared to control group.
There is a significant decrease in blood urea and serum creatinine level in group fed on sucrose plus Pomegranate peel. This result agrees with (Seyedeh et al., 2017) who found that, the supplementation with pomegranate peel extracted exhibited on antihyperlipedemic action, reduced the lipid peroxidation process and enhanced the antioxidant defense system. Moreover, there was significant decrease blood glucose level in groups fed on sucrose plus olive leaves and sucrose plus Pomegranate peel in compared to control group. This result may attributed to the hypoglycemic effect of Pomegranate peel which is confirmed by (Maha, 2017) who mentioned that, in streptozotocin (STZ)induced diabetic rats, Pomegranate peel extract decreases serum concentrations of glucose implying that Pomegranate peel extract is more effective than glibenclamide and may be of use as an antidiabetic agent.

The significant increase in testosterone level in group fed on high sucrose diet. This increment may contribute to increase caloric consumption which has been shown to elevate testosterone level, while decrease caloric intake depresses it (El-Tabakh, 2015).

The obtained data belonging to the effect of Pomegranate peel on testosterone concentration nearly agree with those obtained by El- Gharawi, (2018) who found that pomegranate peel extract significantly decreased serum testosterone as well as sperm count, sperm motility, the weight of prostate, testis, epididymis, seminal vesicle, weights of the testicle and seminal vesicle. Moreover, Pomegranate peel is known as a phytoestrogen plant compound since it contains Lignans and phenolic compounds (Lan et al, 2009). Also, (Maha 2017) reported that, the Pomegranate peel is phytoestrogen which may has an inhibitory effect on the enzyme 17B-hydroxy steroid hydrogenase; 
therefore, the synthesis of testosterone in adrenal cortex is reduced.

The significant decrease in groups fed on sucrose plus Pomegranate peel may attributed to hypocholesterolemic effect of Pomegranate peel according to (Alirezaei et al., 2011) which may be decrease the testosterone testicular synthesis. L'opezMiranda et al., (2006) reported that, the inhibition of de-novo synthesis pathway of cholesterol biosynthesis negatively affects testosterone level in addition to cholesterol concentration in the tissues, body weight gain and ALT with no successful compensatory mechanism as related with testosterone level.

The obtained results revealed that, high sucrose diet induce hyperlipidemia at all periods of the experiment. Thus hyperlipidemia decrease serum zinc concentration according to Ramalingam and Subrahmanyam (2012) who found that, serum zinc levels significantly reduced in high fat fed rabbits than the control group.

The recorded results also revealed that, Pomegranate peel treatment did not alleviate zinc deficiency in groups (3 and 4) when compared to group fed on sucrose only. This may be attributed to potent antioxidant effect of Pomegranate peel that prevent zinc depletion in oxidative process occurred in hyperlipidemic state, this is supported by Maha (2017) who reported that, the positive impact of treatment with crude Pomegranate peel on the antioxidant enzymes GPx, GRx, CAT and SOD observed could be explained with two possible mechanisms. First, the antioxidative effect of Pomegranate peel may prevent further glycosylation and peroxidation of proteins by interacting with free radicals and hence minimizing their noxious effects. Second, Pomegranate peel may induce protein synthesis of these enzymes that explains the observed elevated activity after treatment.
Adekunle et al., (2013) reported that an increase in serum and tissue concentrations of lipids was accompanied by elevated serum copper concentration. Moreover, the increased concentration of lipid peroxides (Malondialdehyde) which is an index of oxidative stress was found in rats given atherogenic diet. There is evidence that copper and zinc have pro-oxidant and antioxidant properties, respectively, so that their imbalance may be expected to condition oxidative stress status (Guo and Wang, 2013). So, the significant decrease in serum copper level in groups kept on high sucrose diet plus Pomegranate peel when compared with group fed on high sucrose diet only may be attributed to Pomegranate peel is hypolipidemic and antioxidant effect.

\section{CONCLUSION}

The findings of the present study demonstrated that pomegranate provided an effective treatment of hyperlipidemia due to sucrose induced stress in rabbits, since this compound was able to ameliorate serum biochemical parameters. We recommended that, we can take advantage of the great therapeutic effects of pomegranate by its administration for patients suffering from hyperlipidemia.

\section{REFERENCES}

Abe A., Yamashita S., Noma A., (1989).Clin. Chem.; 35: 552- 554.

Adekunle A.S., Adedeji A.L., Oyewo E.O., Adedosu O.T., Omotoso A.T. (2013). Hyperlipidemia induced by atherogenic diet enhanced oxidative stress in the kidney and inflammatory responses: an in-vivo study Asian Journal Of Natural \& Applied Sciences 2:(1).

Alirezaei, M; Jelodar, G; Niknam, P; Ghayemi, Z and Nazifi, S (2011). 
Betaine prevents ethanol-induced oxidative stress and reduces total homocysteine in the rat cerebellum. J. Physiol. Biochem., 67: 605-612.

Alonso A, Ruiz-Gutierrez V, MartínezGonzález MA (2006). Monounsaturated fatty acids, olive oil and blood pressure: epidemiological, clinical and experimental evidence. Public Health Nutr 9: 251-257.

Anuradha CVand Anurag P, (2002). Metformin improves lipid metabolism and attenuates lipid peroxidation in high fructose-fed rats. Diabetes ObesMetab 4: $36-42$.

Bauer.J.D., (1982). Clinic laboratory methods. 9 th Ed: 555, the C.V. company, Westline Industrial Missouri.

Bialonska, D., Ramnani, P., Kasimsetty, S.G., Munttha, K.R., Gibson, G.R. and Ferreria, D., (2010): The influnece of pomegranate by product and punicalagins on selected groups of human intestinal microbiota .Int $\mathbf{J}$ food microbial .140, 175-82.

Bray GA (2010). Soft drink consumption and obesity: it is all about fructose. Curr Opin Lipido 1.

Burstein, M.; Selvinick, H.R. and Morfin, R. (1970): Rapid method for the isolation of lipoproteins from human serum by precipitation with polyanions. J. Lipid Res., 11: 583-595.

Busserolles, J.; Rock, E.; Gueux, E.; Mazur, A.; Grolier, P.; Rayssiguier, Y. (2002b). Short term consumption of high sucrose diet has a pro-oxidant effect in rats. Br. J. Nutr., 87, 337- 379.
Caraway, W.T. and Watts, N. B .3ry ed. Philadephia WB sande-rs. 422-447. (1987).

Dobiásová.M, (2004). Atherogenic index of plasma triglyceride/HDL-Cholesterol: Theoretical and practical implications. Clin. Chem.;50: 1113-15.

El-Gharawi H. (2018). Biochemical effect of pomegranate on experimental obesity in rats. MVMsc. thesis, fac. vet. Med. Damanhour Univ. Biocemistry Department.

El-Tabbakh, I, M (2014): Effect of Olive Leaves and Oil on Some Biochemical Constituents in Rabbits Fed On High Sucrose Ration. PhD Thesis in Biochemistry Fac. Vet. Med.e, Alexandria University

Friedewald.W.T., Levy.R.I., Fredrickson.D.S., (1972). Estimation of the concentration of low-density lipoprotein cholesterol in plasma, without use of the preparative ultracentrifuge. Clin Chem; 18: 499502.

Gross LS, Li L, Ford ES, Liu S. (2004) Increased consumption of refined carbohydrates and the epidemic of type 2 diabetes in the United States: an ecologic assessment. Am J Clin Nutr 79: 774-779.

Guo, C. H. \& Wang, C. L. (2013). Effects of Zinc Supplementation on Plasma Copper/Zinc Ratios, Oxidative Stress, and Immunological Status in Hemodialysis Patient. Int J Med Sci, 10(1), 79-89.

Healthy Sugar Alternatives (2012). Organic Lifestyle Magazine. Retrieved -07-20. 307-311. 
Indira P, K., Sujata Khopde, M., SantoshKumar, S. and Hari, Mohan., (2000):Free Radical Studies of Ellagic Acid, a Natural Phenolic Antioxidant .2200-2206.

Johnsen and R. Eliasson (1987). Evaluation of acommercially available kit for the colorimetric determination of zinc. International Journal of Andrology, April 10 (2): 435- 440.

Johnson RJ, Sanchez-Lozada LG, Nakagawa T: (2010). The effect of fructose on renal biology and disease. $\mathrm{J}$ Am Soc Nephrol, 21: 2036-2039.

Kamari Y, Harari A, Shahish A, Peleg E, Sharabi Y, Harats D, and Grossman E(2008) .Hypertens Res 31,135-140.

Kaplan, A., et al., 1984. Bilirubin. Clin Chem The C.V. Mosby Co. St Louis. Toronto. Princeton 1984; 1238-1241, 436 and 650.

L'opez-Miranda J, Badim'on L, Bonanome A, Lairon D, Kris-Etherton PM, Mata P, et al. (2006) Monounsaturated fat and cardiovascular risk. Nutr Rev; 64(s2): s $2-12$.

Lan., Lei, F., Hua, L., Wang, Y., Xing, D. and $\mathrm{Du}$, L.(2009): transport behavior of ellagic acid of pomegranate leaf tannins and its correlation with total cholesterol alteration in HepG2 cells. Biomed chormatogr . 23, $531-6$.

Lansky, EP. and Newman, R.A., ( 2007): Punica granatum (pomegranate) and its potential for prevention and treatment of inflammation and cancer. $J$ Ethnopharmacol. 109,117-206.

Lee, S.I., Kim, B.S., Kim, K.S., Lee, S., Shin, K.S. and Lim, J.S., (2008): ImmuneSuppressive Activity of Punicalagin of
NFAT Activation. Biochemical and Biophysical Research Communications. 371, 799-803.

Lei, F., Zhang, X.N., Wang, W., Xing, D.M., Xie, W.D. and Su, H. (2007): Evidence of anti- obesity effects of the pomegranates leaf extract in high-fat diet induced obese mice. Int $\mathbf{J}$ Obes (Lond). 31, 1023-9.

Litherland GJ, Hajduch E, Gould GW, Hundal HS: (2004). Fructose transport and metabolism in adipose tissue of Zucker rats: diminished GLUT5 activity during obesity and insulin resistance. Mol Cell Biochem, 261:23-33.

Maha,H, El-Moghazy (2017): "pomegranates leaf in Obese diabetic rats and insulin resistance" Master degree in Biochemical dep. Mansoura Univ

Maurya S.K; and Srivastava A. K; ( 2008).High fructose diet-induced glucose intolerance and dyslipidemia in adult Syrian golden hamsters. Indian Journal of Science and Technology; Vol.1 No 6.

Paniagua JA, Gallego de la Sacristana A, Romero I, Vidal- Puig A, Latre JM, Sanchez E, Perez-Martinez P, LopezMiranda J, Perez-Jimenez F.(2007 b) Monounsaturated fat-rich diet prevents central body fat distribution and decreases postprandial adiponectin expression induced by a carbohydraterich diet in insulin-resistant subjects. DiabetesCare; 30: 1717-1723.

Ramalingam K. and Subrahmanyam G., (2012). Serum zinc status, oxidative stress \& chronic low grade inflammation in high fat diet fed rabbits. International Journal of Analytical, Pharmaceutical and 
Biomedical Sciences Page: 13 Volume:

1: Issue-2: April-June-2012 ISSN: 2278-0246

Rommerts, F.F.G. (2004). Testosterone: an overview of biosynthesis, transport, metabolism and non-genomic actions. In: Nieschlag, E., Behre, H.M. eds. Testosterone action, deficiency, substitution, 3rd edition. Cambridge, Cambridge University Press, 1-38.

Rosenblat, M., Hayek, T. and Aviram, M., (2006): Anti-oxidative effects of pomegranate juice (PJ) consumption by diabetic patients on serum and on macrophages. Atherosclerosis, 87, 363371.

Salwe-Kartik, J., Devender, O., Sachdev., Yogesh, Bahurupi. and Manimekalai, Kumarapopan.,(2015): Evaluation of antidiabetic, hypolipedimic and antioxidant activity of hydroalcoholic exract of leaves an fruit peel of punica granatum in male wistar albino rats. 57 Journal of natural science, Biology and medicine. 6, 165-198.

Sarker, M.M.R. and Gohda, E., (2013): Promotion of anti-keyhole limpet hemocyanin Igm and IgG antibody productions in vitro by red bell pepper extract. J Funct Foods. 5, 1918-26.

Schirmeister, J. Willmann, H. and Kiefer, H. (1984): Colorimetric and Kiriebic method for determination of creatinine. Dtsch. Med. Wschr, 89, 1018.

Searcy, R.L., Reardon, J.E., Foreman, J.A. and Amer, J. (1969): Colorimetric method for determination of urea.Med. Techn., 33: 15-20.

Seyedeh, Taheri-Rouhi, Moklesur, R.S., Asmah Rahmat, S., Ahmed, A., and Fauziah, O., (2017). The effect of pomegranate fresh juice versus Pomegranate seed powder on metabolic indices, lipid profile, inflammatory biomarkers, and the histopathology of pancreatic islets of Langerhans in streptozocin-nicotinamide induced type 2 diabetic Sprague-Dawley rats. MBC Complementary and Alternative Medicine 22- 17:156.

Stanhope KL, Griffen SC, Bair BR, Swarbrick MM, Keim NL, Havel PJ. (2008).Twenty-four-hour endocrine and metabolic profiles following consumption of high-fructose corn syrup-, sucrose-, fructose-, and glucose-sweetened beverages with meals. Am J Clin Nutr; 87:1194-203.

Stein.E.A., (1987). Lipids, Lipoproteins and apolipoproteins. Fundementals of clinic chem.3rd Ed: 448-4481. Philadelphia: WB Saunders.

Suzuki T, Hara H: (2004). Ingestion of guar gum hydrolysate, a soluble and fermentable nondigestible saccharide, improves glucose intolerance and prevents hypertriglyceridemia in rats fed fructose. J Nutr 134:1942-1947.

Watson KE, Horowitz BN, Matson G(2003). Lipid abnormalities in insulin resistant states. Rev Cardiovasc Med 4:228-236.

Zöllner N.,Kirsch K;Z. (1962) J.exp.Med., 135, 545. Cited in Biosystem kits for determination of total lipids concentrations 